\title{
El uso de herramientas digitales como videos y blogs para fomentar el aprendizaje de la lengua oral en ELE: un estudio de las percepciones de estudiantes principiantes
}

\author{
Marta Giralt \\ Universidad de Limerick \\ marta.giralt@ul.ie
}

Antonia Flores Universidad de Maynooth antonia.flores@mu.ie

Anna Laribal Universidad de Maynooth anna.laribal@mu.ie

\section{Resumen}

El aprendizaje de la lengua oral por estudiantes universitarios principiantes es un tema de vital importancia desde el punto de vista de la práctica oral, de la toma de conciencia de la pronunciación y del fomento de la confianza y de la autoestima. Sin embargo, la práctica oral no suele estar integrada en clase debido al gran número de estudiantes y a la carga de contenidos que deben cubrirse en muy pocas horas lectivas. En este artículo se presentan datos de un proyecto que tiene como objetivo explorar la inclusión de herramientas digitales para la práctica oral en cursos presenciales. Tras una breve revisión de diferentes estudios que se han realizado sobre este tema, se presentan datos de los participantes en nuestra investigación: 43 alumnos universitarios (Nivel A1), los cuales grabaron un video con información personal. Posteriormente los videos se colgaron en un blog donde los estudiantes dejaron sus comentarios. Después de la tarea, los participantes completaron un cuestionario anónimo sobre aspectos como: el uso de la tecnología, la utilidad de grabar un video y las ventajas del uso de herramientas digitales en el aprendizaje de la lengua oral y de la pronunciación. Los resultados obtenidos nos permiten presentar conclusiones, desde la percepción del alumno, sobre 
los aspectos y factores que intervienen en el aprendizaje de la lengua oral a través de herramientas digitales.

Palabras clave: Lengua oral; pronunciación; vídeo; herramientas digitales.

\title{
Resumo
}

A aprendizagem da língua oral por estudantes universitários principiantes é um tema de vital importância desde o ponto de vista da prática oral, da tomada de consciência da pronunciação e da promoção da confiança e da autoestima. Contudo, a prática oral não costuma estar integrada na prática docente em sala de aula devido ao grande número de estudantes e a carga de conteúdo que deve ser coberta em poucas horas de ensino. Neste artigo se apresentam os dados de um projeto que tem como objetivo explorar a inclusão de ferramentas digitais para a prática oral em cursos presenciais. Após uma breve revisão dos diferentes estudos realizados sobre o assunto, são apresentados dados dos participantes de nossa pesquisa: 43 universitários (Nível A1), que gravaram um vídeo com informações pessoais. Posteriormente, os vídeos foram postados em um blog onde os alunos fizeram seus comentários. Após a tarefa, os estudantes completaram um questionário anônimo sobre aspectos tais como: o uso da tecnologia, a utilidade de gravar um vídeo e as vantagens do uso de ferramentas digitais para aprendizagem da língua oral e da pronunciação. Os resultados obtidos permitem apresentar conclusões, desde a percepção dos alunos, sobre quais aspectos e fatores intervém na aprendizagem da língua oral através de ferramentas digitais.

Palavras-chave: Língua oral; pronunciação; vídeo; ferramentas digitais.

\begin{abstract}
Oral language learning amongst beginner level University students is a topic of vital importance from the point of view of the oral practice, becoming familiar with the pronunciation and the growing confidence and self-esteem. However, oral practice is not usually integrated into class due to the large number of students and the course contents that must be covered in very little time. In this article we present data of a project that had as an objective the exploration of the use of digital tools for in-class oral practice. After a brief literature review, the article presents data from the study participants: 43 University undergraduates (level A1), who recorded a video with personal information. The videos were posted afterwards in a blog where the students left their comments. After the task, the learners completed an anonymous questionnaire relating to different aspects such as: the use of technology, the practicality of recording a video and the advantages of using digital resources in the learning of a language orally and the pronunciation. The results obtained allowed us to present our conclusions, from the student perception
\end{abstract}


regarding the different aspects and factors that intervene in the learning of oral language through the use of digital tools.

Keywords: Oral language; pronunciation; video; digital tools.

\section{Introducción y justificación del estudio}

La relevancia de la expresión oral en el desarrollo de la competencia comunicativa es un aspecto indiscutible, como bien han señalado los investigadores que se ocupan del estudio de la oralidad (entre ellos, BARALO, 2000; PINILLA, 2000; AGUSTÍN LLACH, 2006).

A pesar de ello, el papel que se le otorga a la expresión oral en la enseñanza superior de segundas lenguas no siempre es el deseado debido en ocasiones a la propia estructuración de los cursos, al alto número de estudiantes o a la falta de tiempo, y, en muchos casos, queda relegada a un papel secundario. Así, en muchas instituciones universitarias, la expresión oral es trabajada tan solo unos minutos durante las clases de lengua general, en otras ocasiones, es asignada totalmente a las clases de conversación (si estas existen) o a las clases de laboratorio (donde principalmente se trabaja la comprensión oral).

Muchos docentes de la enseñanza superior coincidimos en que las clases de lengua necesitan más práctica oral y entendemos que la práctica de la lengua oral y la pronunciación deben estar integradas en el discurso oral (nivel segmental y suprasegmental) para desarrollar la adquisición de las competencias fónicas y con ello la competencia comunicativa (CANTERO, 2011).

Partiendo de estas premisas, desarrollamos el siguiente proyecto dirigido a nuestros estudiantes principiantes y que consistió en la preparación de grabaciones de video hechas por los propios estudiantes a partir de instrucciones dadas con el objetivo no solo de fomentar el desarrollo de la expresión oral, sino también observar cómo estas grabaciones pueden ser herramientas en el desarrollo de la pronunciación, la entonación $\mathrm{u}$ otros aspectos relevantes en el aprendizaje de la lengua oral como son los aspectos afectivos.

El uso de recursos audiovisuales para el aprendizaje del español resulta ideal gracias a su gran potencial didáctico, creativo y motivador dentro de un enfoque comunicativo y de una pedagogía 
multimodal en la clase de ELE: modo lingüístico, visual, auditivo, gestual, espacial, pragmático y sociocultural.

Trabajar con material audiovisual creado por los propios alumnos en el aula les motiva ya que estos se sienten protagonistas de su trabajo y tienen un papel más activo durante el proceso, al igual que les permite auto-reflexionar sobre su producto final con el autovisionado. Con las grabaciones, la producción oral se desliga de la preponderancia que presenta el contenido en una comunicación oral real, no se centra fundamentalmente en la transmisión del mensaje, sino que se le da una mayor relevancia a la forma y a la manera que se transmite el mensaje.

Teniendo todas estas cuestiones en cuenta, las preguntas que nos planteamos al iniciar este estudio fueron las siguientes:

1) Desde la perspectiva y percepción de estudiantes principiantes, ¿resultaría beneficioso/útil la grabación de un video para el aprendizaje de la lengua oral y la pronunciación? ¿En qué medida? ¿Qué factores intervienen?

2) ¿Cómo perciben los estudiantes el uso del video frente al audio?¿Qué ventajas existen?

3) ¿Cómo perciben que los videos se compartan en un blog entre todos los estudiantes? ¿Qué aspectos positivos aportan?

Tras establecer las preguntas, presentamos ahora una revisión de los estudios e investigaciones existentes en el campo de la enseñanza y aprendizaje de la lengua oral y la pronunciación mediados por ordenador puesto que nuestra investigación se enmarca en este.

\section{Revisión de los estudios sobre la enseñanza y aprendizaje de la lengua oral y la pronunciación mediados por ordenador}

El aprendizaje de la lengua oral y la pronunciación asistido por ordenador ha sido foco de atención en los últimos años. Varios son los estudios que abordan el tema, tanto en el ámbito de la enseñanza y aprendizaje del inglés como en el del español como lengua extranjera. Desde el punto de vista de la enseñanza y aprendizaje de la pronunciación y la percepción auditiva mediada por ordenador, existen estudios sobre el uso de determinados softwares para el aprendizaje de la pronunciación (HINCKS, 2003; PI-HUA, 2006; CABEDO, 2012; 
HANNA, GAO, 2016) o sobre métodos instruccionales asistidos por ordenador para la enseñanza y aprendizaje de la pronunciación y la entonación (MOLHOLT, 1988; CHUN, 1989; HARDISON, 2004; LLISTERRI, 2007).

Asimismo se han llevado a cabo estudios en los que se usan herramientas digitales tales como los podcasts (CHACÓN, PÉREZ 2011; FACER, ABDOUS, CAMARENA, 2009; ABDOUS, CAMARENA, FACER, 2009) para favorecer el aprendizaje de la comprensión y producción de la lengua oral, los audioblogs para apoyar el aprendizaje de la lengua oral (HSU, WANG, COMAC, 2008) o la aplicación de un enfoque oral digital en el que la enseñanza de la pronunciación y la lengua oral se realiza únicamente a través de la oralidad y con el soporte de herramientas digitales (SPALACCI, 2014).

La utilización de la Web 2.0 para la enseñanza y aprendizaje de la pronunciación también ha sido objeto de estudio, y en este área trabajos como los de Tomé (2010) proporcionan una panorámica muy completa de las distintas posibilidades que esta web puede ofrecer.

Nuestro estudio se basa en la utilización de videos y de herramientas de la Web 2.0, como son los blogs, y al igual que el estudio presentado por Tomé, trabajamos también con alumnos principiantes. Cabe mencionar además, la literatura que existe sobre el uso de voice board para la enseñanza de la lengua oral en inglés (SONG, 2009) o de video blogging (HUNG, DANNY HUANG, 2015). En el campo del español, son muy pocos los trabajos que se pueden encontrar en esta línea. La mayoría de estudios en los que se utiliza video para el aprendizaje de la lengua oral se abordan desde la perspectiva del uso de materiales audiovisuales con incidencia en la comprensión oral más que en la producción (CASÁN, 2009), en el uso de materiales audiovisuales como herramienta pedagógica, especialmente el cine, para aprender español (LAKSFOSS, 2009; TORO, 2009; GIRALT, 2011) o la creación de cortos o materiales audiovisuales en el aula (CAÑADILLAS, 2012).

Así, con nuestro trabajo pretendemos contribuir a expandir el estudio de la enseñanza y aprendizaje mediado por ordenador de la lengua oral y la pronunciación en ELE, basándonos en la creación de videos o audiovisuales para explorar qué repercusión tiene en los estudiantes y cómo es percibido por ellos. 


\section{Descripción de nuestro proyecto}

El proyecto recibe el nombre de Blended Learning Pedagogy in Second Language Teaching: developing oral language skills in Spanish y así se registró una vez obtuvo la aprobación del "Research Ethics Committee" de la Universidad de Maynooth, Irlanda. Este proyecto, como ya hemos mencionado, explora la inclusión de herramientas digitales (video y blog) para la práctica de la lengua oral en cursos presenciales (de nivel principiante). Este acercamiento mixto permite que los alumnos practiquen la lengua oral fuera del aula, al mismo tiempo que forma parte del módulo de lengua que están cursando.

Los participantes involucrados en este proyecto eran 43 estudiantes universitarios irlandeses e internacionales de Erasmus (procedentes de Alemania, Estados Unidos, Francia, Italia, Lituania y Polonia) que eran aprendices principiantes sin previo conocimiento de español. Estos estudiantes cursaban español como parte de su grado doble con diferentes especializaciones.

Los estudiantes que participaron en este proyecto estaban cursando el módulo de "Spanish for Beginners" ofrecido como asignatura obligatoria de primer año dentro del Departamento de Español y Estudios Latinoamericanos que forma parte de la School of Modern Languages, Cultures and Literature en la Universidad de Maynooth (Irlanda). El módulo consistía en 78 horas totales, repartidas en 6 horas y media semanales distribuidas a lo largo de las 12 semanas del semestre entre los meses de septiembre y diciembre.

Para la intervención didáctica con esta actividad se tuvieron en cuenta los contenidos ya trabajados y los que queríamos que plasmaran en la actividad, la cual constaba de dos partes:

1) Creación del video

2) Realización del Treasure Hunt.

En la primera parte de la actividad, los estudiantes tenían que grabar un video en la semana 10 del curso hablando en español y ofreciendo información personal y describiendo su físico, sus gustos y aficiones, su familia, su pueblo, sus estudios, etc.

Los estudiantes disponían de libertad absoluta en su creación y originalidad, asegurándose de incluir la información pedida, no exceder los 5 minutos y seguir unos códigos de conducta obvios. 
Posteriormente, todos los videos de los estudiantes se colgaron en un blog de clase para que estos los pudieran ver, dejar comentarios y hacerse preguntas.

Cabe destacar que el blog era totalmente privado, sólo los estudiantes de esa clase, la profesora y la coordinadora de primer año tenían acceso a él. Previamente, los estudiantes firmaron un documento para dar su consentimiento legal para subir los videos al blog y usarlos sólo con finalidades educativas.

Para la grabación del video se utilizó la plataforma Voicethread y Vimeo, que ofrece la posibilidad de grabar imagen y sonido. Los estudiantes fueron guiados mediante un documento con instrucciones muy concretas y todos los pasos a seguir para que la grabación del video les resultará sencilla y práctica. Además, cada profesora hizo una muestra en clase donde tuvieron la oportunidad de preguntar cualquier duda y posteriormente, contaron con el apoyo de la coordinadora que les ayudó en la grabación cuando lo necesitaron.

En la segunda parte de la actividad, los estudiantes tenían que realizar un Treasure Hunt en el que, primero necesitaban visionar los videos de sus compañeros y, después, contestaban preguntas como: ¿Quién es de Dublín? ¿A quién le gusta la música rock? ¿Quién almuerza a la 1.30? ¿Quién tiene dos hermanos? (Véase Figura 1)

Tras la finalización de la tarea global, los estudiantes completaron un cuestionario anónimo (véase apartado 4 de este artículo).

La integración de esta actividad dentro del módulo nos resultó sencilla. La creación del video se planteó como una actividad obligatoria integrada como parte de su evaluación formativa. La calificación de dicha actividad quedó contemplada dentro de un 3\% como una de sus 5 tareas de CA (continuous assessment) que realizan a lo largo del primer semestre. En cambio, la participación en la segunda parte de la actividad, Treasure Hunt, se planteó como una actividad no evaluable sino voluntaria.

\section{Metodología}

La metodología de la investigación se basa en los datos recogidos a partir de un cuestionario virtual en el que se pedía a los 
estudiantes que contestaran distintas preguntas sobre aspectos tales como: el uso de la tecnología, la utilidad de grabar un video para el aprendizaje de la lengua oral o las ventajas del uso de herramientas digitales para el aprendizaje de la pronunciación. No solo nos interesaba ver los resultados de los videos para sacar conclusiones de su eficacia a nivel de la producción oral sino que también queríamos saber qué pensaban los estudiantes para poder analizar profundamente el impacto del proyecto y cómo estos lo habían percibido.

Una vez finalizado el semestre se distribuyó el cuestionario a través de un enlace que les llegó a su correo personal, para que lo contestaran de un modo virtual (más cercano/atractivo a su código), libre (cuándo y dónde quisieran) y anónimo (total libertad de expresión). De este modo los estudiantes nos pudieron transmitir sus percepciones sobre la actividad de una manera más relajada y objetiva.

Figura 01 - Muestra de uno de los blogs de clase con los enlaces a los videos de los estudiantes y el Treasure Hunt

\section{Yo en español_Marta}
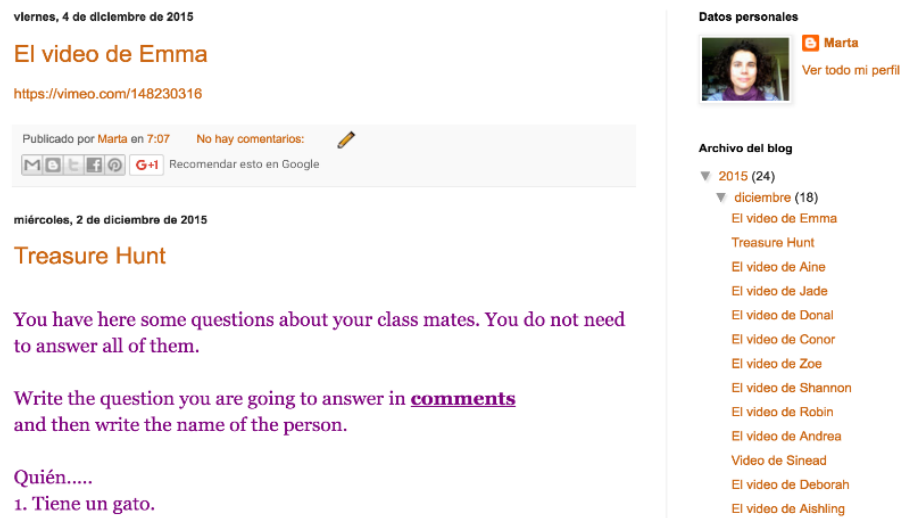

Archivo del blog

V 2015 (24)

v diciembre (18)

El video de Emma

Treasure Hunt

El video de Aine

El video de Jade

El video de Donal

El video de Conor

El video de Zoe

El video de Shannon

El video de Robin

El video de Andrea

Video de Sinead

El video de Deborah

El video de Aishling

El cuestionario constaba de 9 preguntas directas y una pregunta que se desdoblaba en otra a partir de la respuesta dada por el alumno. Del total de estas 10 preguntas, tres de ellas (preguntas 1, 2 y 7) seguían una escala de puntuación del 1 al 5 en la que los estudiantes 
tenían que marcar su preferencia. También se usaban preguntas basadas en una respuesta excluyente en la que los estudiantes debían escoger entre "Yes" y "No" (preguntas 6, 8); "Yes", "No", "Maybe" (pregunta 9) o "Yes", "No", "Yes, I went back and realised that I have improved" (pregunta 4).

Junto a estos dos tipos de preguntas, el cuestionario constaba también de preguntas en las que se daba opcionalidad de respuestas y los alumnos podían marcar más de una (preguntas 3 y 5). Cabe mencionar que además, tres de las preguntas del cuestionario (preguntas 8, 5 y 3) ofrecían también la posibilidad de una respuesta abierta con la opción "Other".

Respecto al contenido de las preguntas, el primer aspecto que exploramos con las cuestiones 1 y 2 (How did you find to record a video speaking in Spanish for your Spanish class?; Do you think it was a useful activity for developing your oral language skills in Spanish?) fue de qué manera percibieron los estudiantes la experiencia de grabar un video, y si la actividad les resultó positiva o negativa. Asimismo, se les pidió evaluar si la tarea les había resultado beneficiosa para mejorar y desarrollar sus destrezas orales.

Con las preguntas número 3 y 4 (How did the activity help you on your Spanish pronunciation and your oral language skills?; Did you go back to your video to listen to yourself and learn from your mistakes?) pretendíamos que los alumnos reflexionaran sobre cómo esta actividad les había ayudado con la familiarización y pronunciación de ciertos sonidos, la toma de confianza a la hora de hablar y la toma de conciencia de sus habilidades orales. Asimismo, los alumnos reflexionaron sobre las posibles ventajas que lleva consigo el autovisionado para mejorar aspectos como la pronunciación, los errores gramaticales y la entonación.

Junto a estas primeras percepciones sobre cuestiones estrechamente vinculadas con el aprendizaje de la lengua oral y la pronunciación, la pregunta 5 (Did you find any of these benefits listed below when recording a video instead of an audio?) rastreaba las distintas percepciones que los alumnos pudieran tener sobre el hecho de usar video y no únicamente audio. Los alumnos en este punto reflexionaron sobre qué aspectos multimodales les ayudaron a comunicar su mensaje: aspectos visuales, aspectos gestuales, aspectos espaciales u otros que ellos consideraran. 
Las siguientes dos preguntas, 6. Did you enjoy working with technology as a component of your language module y 7 . Did you find difficult the use of technology?, tenían como objetivo averiguar si los estudiantes disfrutaron utilizando la tecnología como una herramienta más para su aprendizaje de la lengua y conocer las dificultades con las que se habían encontrado.

Dado que la actividad de grabación del video iba acompañada de una tarea de visionado de los videos de los otros compañeros y de un Treasure hunt, con las preguntas 8 y 9 (Did you like listening some of the other students videos for the Treasure Hunt?; If you said yes, why?) se pretendía averiguar si les había gustado ver los videos de sus compañeros y por qué.

Finalmente, la última pregunta del cuestionario, 10. Would you like to do similar activities to practice your Spanish in the future?, tenía como objetivo saber si a los alumnos les gustaría volver a realizar este tipo de actividades en el futuro para mejorar su aprendizaje del español.

\section{Resultados y Discusión}

Tras el análisis de los cuestionarios, se han extraído resultados que reflejan la percepción que los estudiantes tuvieron respecto al uso de herramientas digitales para el aprendizaje de la lengua oral. A continuación comentaremos cada uno de los resultados analizados y discutiremos qué relación puede establecerse entre los procesos de aprendizaje de la lengua oral y de qué forma parecen percibirlos los estudiantes.

A la mayoría de los alumnos les gustó realizar la grabación de video y además encontraron que había sido una actividad muy beneficiosa para el desarrollo de sus destrezas orales, como puede apreciarse en los gráficos 1 y 2, donde la gran totalidad de respuestas se sitúan en la parte positiva de la escala de valores del 1 al 5. Además, el $76,7 \%$ de los estudiantes contestaron que habían disfrutado usando tecnología como parte de su módulo de lengua frente a un 23,3\% que contestó que no. 
Figura 02 - Percepción general de los estudiantes sobre la grabación del vídeo

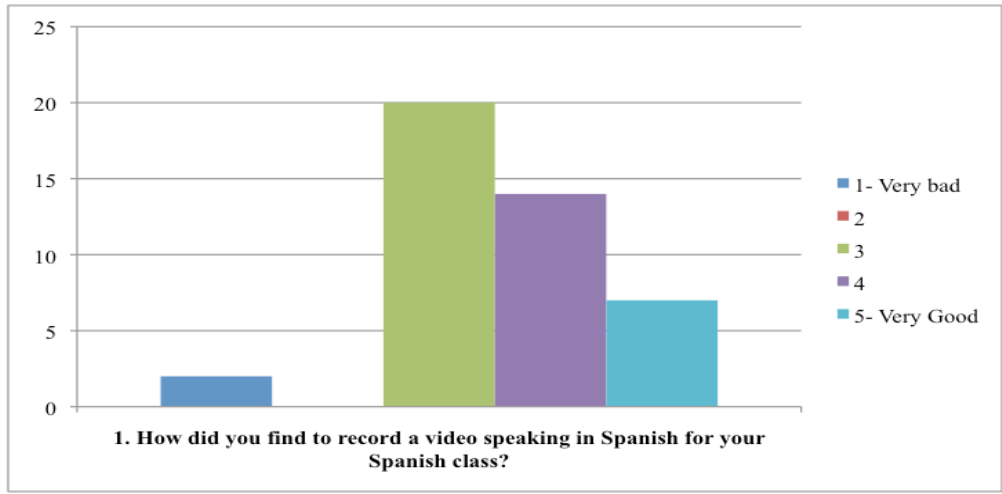

Estos primeros resultados muestran que los alumnos percibieron la realización de la tarea digital como parte de su módulo de lengua como una experiencia positiva y beneficiosa. Todos los alumnos percibieron que la tarea les había ayudado en el aprendizaje de la lengua oral y la pronunciación. Ningún estudiante de los que contestó al cuestionario marcó la opción "Did not help me at all”. Los distintos aspectos que los alumnos percibieron como más relevantes y útiles para el aprendizaje de la lengua oral y la presentación pueden apreciarse en el gráfico 3 .

El hecho de que la tarea fuera una actividad en la que los estudiantes tenían que hablar y podían grabarse tantas veces como les fuera necesario, les ayudó a familiarizarse con la producción oral y la pronunciación una gran variedad de sonidos del español. La familiarización con la producción de algunos sonidos del español fue el aspecto más mencionado por todos los alumnos aunque muchos de ellos también percibieron que la tarea les había ayudado a mejorar la pronunciación de ciertos sonidos. 
El uso de herramientas digitales...

Figura 03 - Percepción de los estudiantes sobre la utilidad de la grabación del video

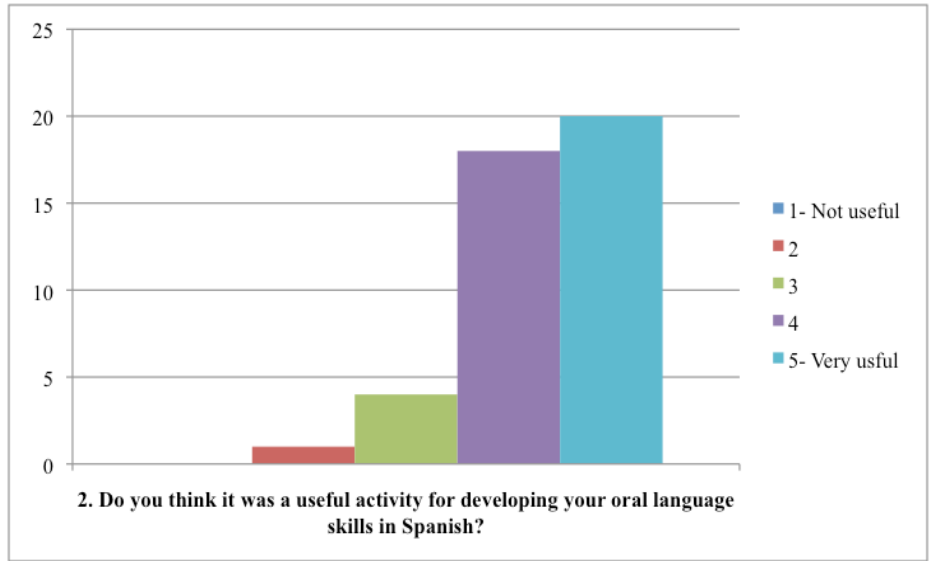

La toma de confianza fue una de las consecuencias más importantes que mayor número de estudiantes percibió tras la realización de la tarea, a diferencia del sentimiento de logro que había supuesto la grabación del video. Los comentarios libres que los alumnos dejaron en esta pregunta fueron solamente dos:

- "Listening back let me hear the mistakes I made especially re fem/masc word endings"

- "I could not concentrate on pronunciation because I was caught up of what to say”

El primer comentario claramente hace referencia a la utilidad que tiene el re-visionado del video, no solo por las cuestiones relacionadas con la pronunciación, como más adelante comentaremos, sino por cuestiones de corrección gramatical. El segundo comentario pone de manifiesto la dificultad que supone para alumnos principiantes poder focalizar en la pronunciación durante la producción oral, y que en cierta manera justifica la eficacia y utilidad de actividades basadas en grabaciones de video donde los alumnos pueden realizar un revisionado. 
Figura 04 - Percepciones de los estudiantes sobre el impacto de la actividad en su pronunciación

3. How did the activity help you on your Spanish pronunciation and your oral language skills?

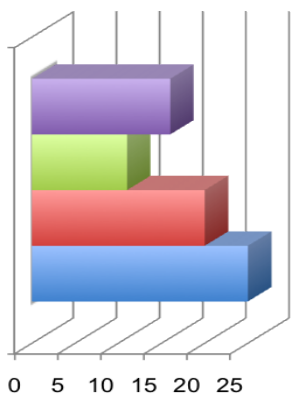

- Helped me to improve pronunciation of certain sounds

- Made me feel proud when listening how much I could say in Spanish

- Made me feel more confident when speaking in Spanish

= Helped me to get familiar with the pronunciation of certain sounds

Durante el desarrollo de la tarea, la mayoría de los estudiantes volvieron a ver sus propias grabaciones y un alto porcentaje puntualizó que esta actividad les había permitido mejorar sus destrezas orales (véase gráfico 4). El auto-visionado les proporcionó la oportunidad de reflexionar sobre su propio aprendizaje.

Asimismo, como puede apreciarse en el gráfico 5, la mayoría de los estudiantes señala haber visto los videos presentados por el resto de la clase para realizar el Treasure Hunt, el cual debían llevar a cabo una vez todos los enlaces de los videos se hubiesen colgado.

En relación a las razones por las que vieron las grabaciones de sus compañeros (véase Gráfico 6), la mayor parte de ellos señaló que el visionado les había permitido ser conscientes de que todos los estudiantes de la clase estaban llevando a cabo un proceso de aprendizaje similar y que todos estaban experimentando dificultades parecidas a las suyas. Este hecho permite entrever cómo los estudiantes están percibiendo su identidad de aprendices de una lengua, identidad que comparten con el grupo clase y que les permite sentirse cómodos cuando cometen errores o se enfrentan a alguna dificultad (PASFIELD-NEOFITOU, 2011). 
El uso de herramientas digitales...

Figura 05 - Porcentajes de los estudiantes que repitieron el visionado del video

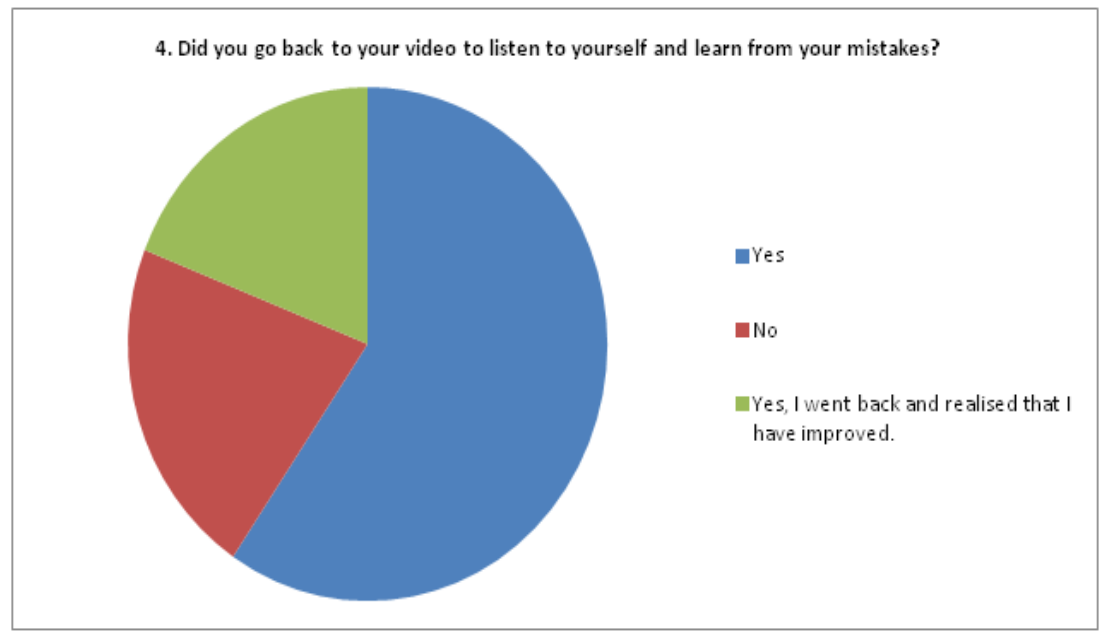

Figura 06 - Opiniones sobre el visionado de videos de otros estudiantes

8. Did you like listening some of the other students videos for the Treasure Hunt?

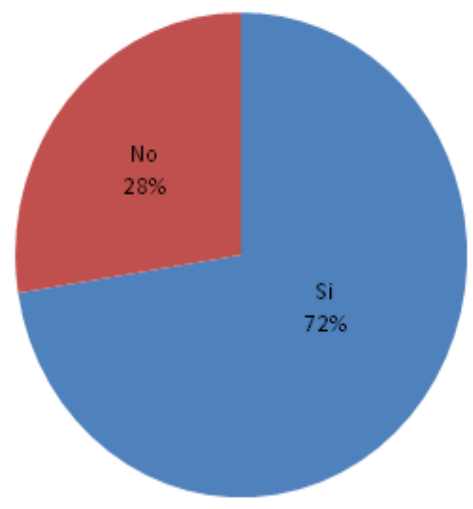

Otro aspecto remarcable es que un alto porcentaje de los participantes admitió que el visionado de las grabaciones de sus 
compañeros les había permitido conocerlos mejor, hecho muy importante al tratarse de alumnos de primer curso de universidad.

Hay que señalar que un porcentaje muy bajo de estudiantes no realizó el visionado de los videos de sus compañeros por falta de tiempo o por sentir que al visionarlos invadía la intimidad de estos. Aquí, nos encontramos con cuestiones relacionadas con la privacidad, un elemento a tener en cuenta cuando se trabaja con herramientas digitales que permiten el acceso de todos los miembros del grupo a las producciones de los alumnos.

Figura 07 - Motivos por los que vieron los videos de otros estudiantes

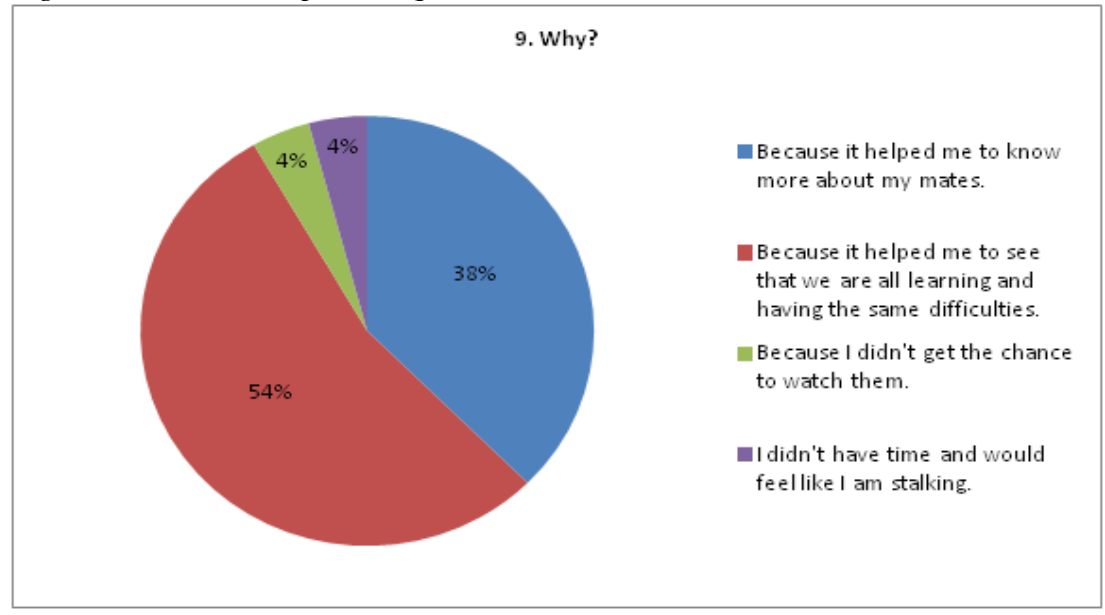

Otro punto muy importante relacionado con la utilización de grabaciones audiovisuales se asienta en la riqueza semiótica que este tipo de medio otorga a la comunicación de los alumnos. El uso de distintos modos semióticos como son los gestos, los movimientos faciales, los colores o el espacio forman parte del entramado multimodal enriqueciendo la comunicación en las producciones orales de los alumnos (ALFONSO, GIRALT, 2014). Así, los alumnos percibieron que el hecho de poder utilizar el lenguaje facial (movimiento de las cejas, sonrisa) les ayudó en la producción oral al utilizar video. Como puede apreciarse en el gráfico 7, el lenguaje facial parece que tiene una función más compensatoria que el lenguaje gestual, mientras que el poder ver la imagen de la persona que está 
hablando en el video es percibido por los alumnos como un factor importante y beneficioso.

A pesar de que la mayoría de los estudiantes percibió que la multimodalidad les había ayudado en sus producciones orales, 12 alumnos sintieron que el hecho de utilizar video frente a audio no suponía ninguna diferencia para ellos.

En los cuestionarios encontramos también un comentario libre de un alumno: "It makes sure that you know what they are saying and not reading from a page", comentario que refleja que el estudiante no percibe la actividad de la grabación en video como una práctica oral de expresión lectora.

En cuanto al uso de herramientas digitales y tecnología, la mayoría de los estudiantes no encontró difícil el uso de la tecnología. En el gráfico 8 se aprecia que 25 de las respuestas de los estudiantes se sitúan en la parte positiva de "fácil" y "muy fácil", mientras que 3 de las respuestas se sitúan en la parte negativa de "difícil" y "muy difícil".

Figura 08 - Percepciones de los beneficios de trabajar con video en vez de audio

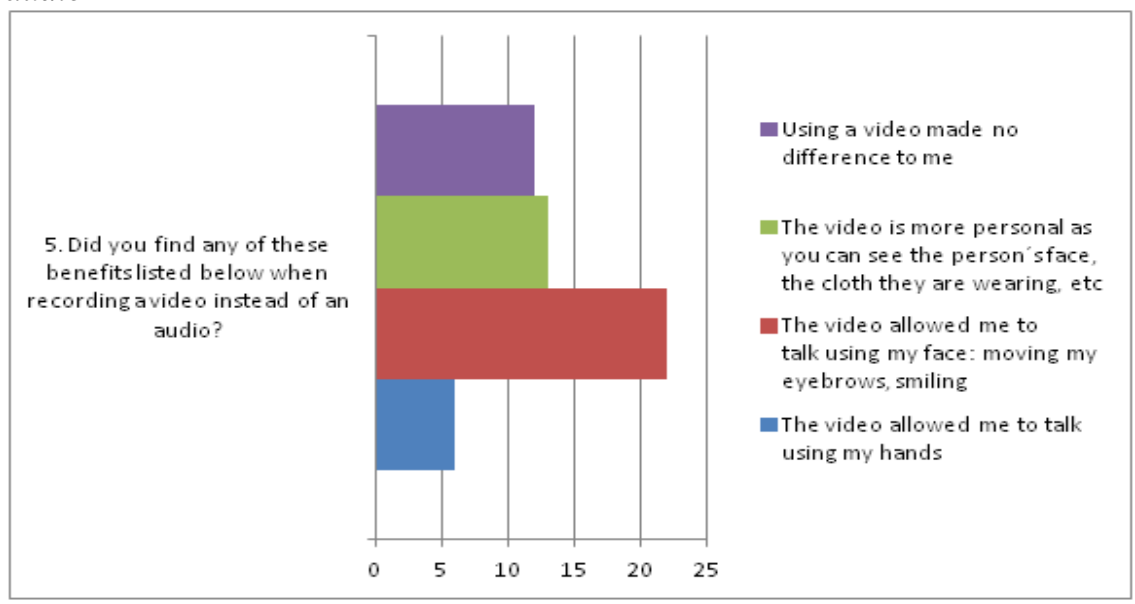

A un $60 \%$ de los estudiantes les gustaría volver a hacer actividades de este tipo en el futuro para practicar y mejorar su español, al mismo tiempo que un $31 \%$ afirma que quizás querrían 
Marta Giralt; Antonia Flores; Anna Laribal repetir esta actividad. Sólo un 9\% parece no querer volver a repetir la actividad.

Figura 09 - Dificultad en el uso de la tecnología

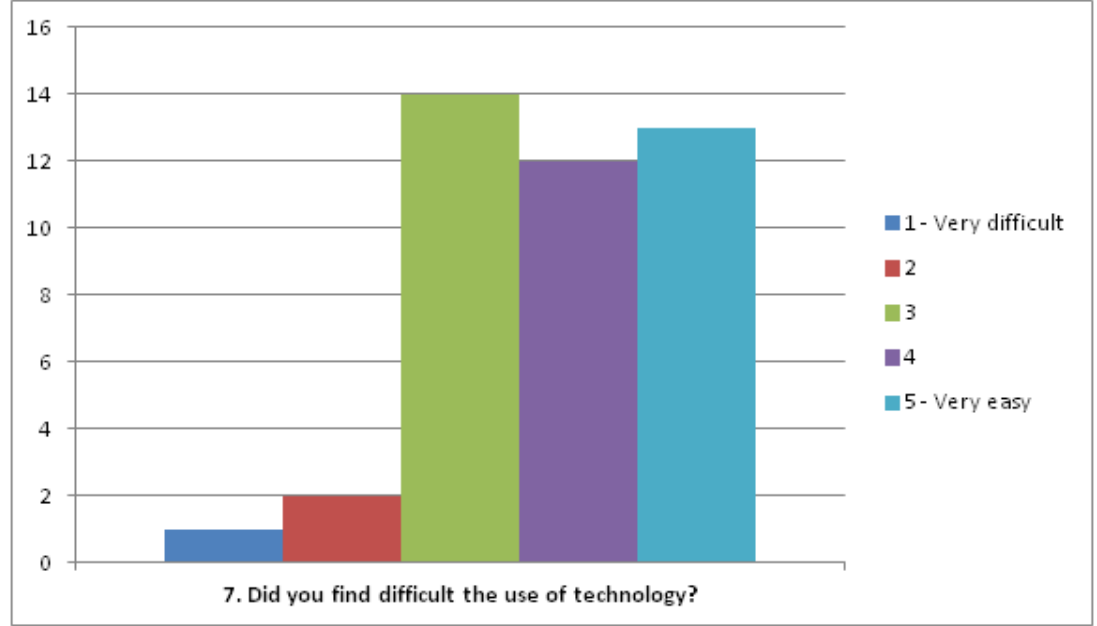

Figura 10 - Porcentajes de los alumnos que repetirían la actividad de la grabación en video

10. Would you like to do similar activities to practice your Spanish in the future?

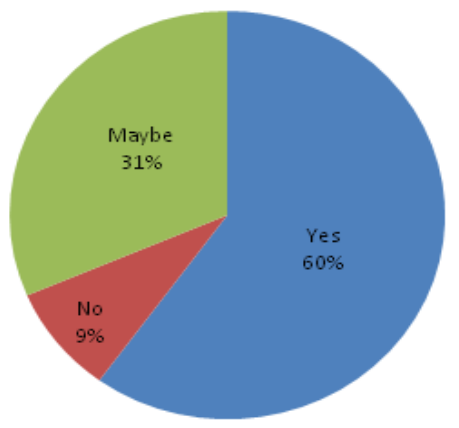




\section{Conclusiones}

La experiencia del uso de videos para el aprendizaje de la lengua oral fue altamente positiva y la implementación del proyecto recibió muy buen feedback por parte de los alumnos. Los resultados sobre el análisis de los cuestionarios y los videos de los alumnos nos han permitido explorar y presentar algunas conclusiones sobre qué aspectos y factores intervienen en el aprendizaje de la lengua oral a través de herramientas digitales, tales como la toma de confianza; la familiarización con la pronunciación y práctica oral o la toma de conciencia de los errores. Asimismo, los datos revelan cómo los alumnos tienden a preferir la grabación de un video a la de un audio puesto que la significación semiótica de imagen y palabras es mucho más amplia, especialmente la significación del lenguaje facial en primer lugar y gestual en segundo lugar.

Tras el éxito de esta primera fase del proyecto, nos gustaría ampliarlo a una segunda fase en la que el mismo proyecto se llevará a cabo en distintas lenguas. A su vez, otras cuestiones que también nos gustaría seguir explorando son la ansiedad que puede provocar la grabación de un video para que otros compañeros de clase lo vean y el análisis multimodal de las producciones audiovisuales de los alumnos.

\section{Referencias}

ABDOUS, M’Hammed; CAMARENA Margaret; FACER, Betty. MALL technology: Use of academic podcasting in the foreign language classroom. ReCall, v. 21, p. 76-95, 2009. Disponível em: $<$ https://pdfs.semanticscholar.org/6e9d/7aeb862fefffd2406100ce1313b dcd3234f1.pdf >. Acesso em: 5 nov. 2019.

AGUSTÍN LLACH, Maria Pilar. La importancia de la lengua oral en la clase de ELE: estudio preliminar de las creencias de aprendices. Actas del XVII Congreso Internacional de la ASELE. 2006. Disponível em:

$<$ https://cvc.cervantes.es/ensenanza/biblioteca_ele/asele/pdf/17/17_016 1.pdf>. Acesso em: 5 nov. 2019. 
ALFONSO, Raul; GIRALT, Marta . Comunicación multimodal y aprendizaje de la lengua oral en E/LE. CAUCE. Revista Internacional de Filología, Comunicación y sus Didácticas, v. 36/37, p. 117-133, 2014. Disponível em: $<$ https://cvc.cervantes.es/literatura/cauce/pdf/cauce36-37/cauce_3637_007.pdf $>$. Acesso em: 5 nov. 2019.

BARALO, Marta. El desarrollo de la expresión oral en el aula de ELE. Carabela, 27, p. 5-36, 2000. Disponível em: $<$ https://www.nebrija.com/revistalinguistica/files/articulosPDF/8.Baralo1.pdf >. Acesso em: 5 nov. 2019.

CABEDO NEBOT, Adrián. Actividades de articulación y de percepción auditiva: uso de herramientas informáticas. Foro de Profesores de E/LE, v. 8, 2012.

CANTERO, F.,J.. Adquisición de competencias fónicas, en Congosto, Y.; Salvador, A.; Montero Curiel, M.L. (Eds.). Fonética experimental. Educación Superior e Investigación. Colección Bibliotheca Philologica. Madrid, Editorial Arco/Libros, 2011. p. 29-55.

CAÑADILLAS, Fernando. Producción audiovisual en el aula de ELE: una propuesta didáctica. Memoria de Máster. Universidad de Oviedo. 2012.

Disponível

em: $<$ http://digibuo.uniovi.es/dspace/bitstream/10651/3933/6/TFM_Fernan do_Ca\%C3\%B1adillas_Ramallo.pdf $>$. Acesso em: 5 nov. 2019.

CASÁN, Juan Carlos. Didáctica de las grabaciones audiovisuales para desarrollar la comprensión oral en el aula de lenguas extranjeras. MarcoELE. Revista de Didáctica Español Lengua Extranjera, (9), 2009, p. 1-14. $\quad$ Disponível em: <https://www.redalyc.org/pdf/921/92152528015.pdf >. Acesso em: 5 nov. 2019.

CHACÓN, Carmen.; PÉREZ, Clevia. El podcast como innovación en la enseñanza del inglés como lengua extranjera. Pixel-Bit, Revista de Medios y Educación, (39), 2011. Disponível em: 
<https://recyt.fecyt.es/index.php/pixel/article/viewFile/61449/37462>. Acesso em: 5 nov. 2019.

CHUN, Dorothy. Teaching tone and intonation with microcomputers, CALICO Journal, 7, 1989. p. 21-46. Disponível em: $<$ http://www1.cs.columbia.edu/ sbenus/Research/L2_Intonation/Chun. pdf>. Acesso em: 5 nov. 2019.

FACER, B; ABDOUS, M.; CAMARENA, M.. The Impact of Academic Podcasting on Students: Learning Outcomes and Study Habits en De Cassia Veiga Marriott, R.; Lupion Torres, P. (Eds.) Handbook of Research on E-Learning Methodologies for Language Acquisition Acquisition. IRM Press: Cybertech Publishing. 2009. p. 339-351.

GIRALT, Marta. El cine como herramienta didáctica y su transversalidad en el aula ELE. Actas del XVIII Seminario de dificultades específicas de la enseñanza del español a lusohablantes, Consejería de Educación en Brasilia, XVIII, 135-158, 2011. Disponível em: <http://mural.maynoothuniversity.ie/6622/1/MG-herramientadid\%C3\%A1ctica.pdf $>$. Acesso em: 5 nov. 2019.

HANNA, Barbara E.; GAO, Yang. Exploring optimal pronunciation teaching: Integrating instructional software into intermediate-level EFL classes in China. Calico journal, v. 33, n. 2, 201, 2016. DOI: 10.1558/cj.v33i2.26054

HARDISON, Debra. Generalization of computer-assisted prosody training: Quantitative and qualitative findings. Language Learning $\boldsymbol{\&}$ Technology, v. 8, 2004, 34-52.

HINCKS, Rebecca. Speech technologies for pronunciation feedback and evaluation, ReCALL, v. 15, n. 1, p. 3-20, 2003. DOI: https://doi.org/10.1017/S0958344003000211

HSU, Hui-Yin; WANG, Shiang-Kwei; COMAC, Linda. Using audioblogs to assist English-language learning: An investigation into 
student perception. Computer Assisted Language Learning, v. 21, n. 2, p.181-198, 2008. DOI: 10.1080/09588220801943775

HUNG, Alan; HUANG, Danny. Video Blogging and English Presentation Performance: A pilot study. 1, 2. Psychological Reports, v. 117, n. 2, 2015. p. 614-630. DOI: 10.2466/11.PR0.117c20z6

LAKSFOSS, Ann. ¡Acción! El uso de materiales audiovisuales en el aula de ELE. Actas de ANPE II Congreso nacional: Multiculturalidad y norma policéntrica: Aplicaciones en el aula de ELE. 2008, p. 1-9. Disponível em: <http://www.educacionyfp.gob.es/dam/jcr:ebf8717f-0b5a-4eae-b9cc-

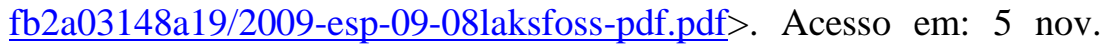
2019.

LLISTERRI, Joaquim. La enseñanza de la pronunciación asistida por ordenador. En Actas del XXIV Congreso Internacional de AESLA. Aprendizaje de lenguas, uso del lenguaje y modelación cognitiva: perspectivas aplicadas. 2007, p. 91-120. Disponível em: $<$ http://liceu.uab.cat/ joaquim/publicacions/Llisterri 06 Pronunciacio n_Tecnologias.pdf>. Acesso em: 5 nov. 2019.

MOLHOLT, Garry. Computer-assisted instruction in pronunciation for Chinese speakers of American English, TESOL Quarterly, 22 (1), 1988, p. 91-111.https://www.learntechlib.org/p/140045/>. Acesso em: 5 nov. 2019.

PASFIELD-NEOFITOU, Sarah. Online domains of language use: Second language learners' experiences of virtual community and foreignness. Language Learning and Technology, v. 15, n. 2, p. 92108, 2011. Disponível em: <http://citeseerx.ist.psu.edu/viewdoc/download?doi=10.1.1.669.6702\& rep=rep1\&type=pdf $>$. Acesso em: 5 nov. 2019.

PI-HUA, Tsai. Bridging pedagogy and technology: User evaluation of pronunciation oriented CALL software. Australasian Journal of Educational Technology, v. 22, n. 3, p. 375-397, 2006. Disponível em: 〈https://doi.org/10.14742/ajet.1292>. Acesso em: 5 nov. 2019. 
PINILLA, Raquel. El desarrollo de las estrategias de comunicación en los procesos de expresión oral: un recurso para los estudiantes de ELE, Carabela, v. 27, p. 53-68, 2000. Disponível em: <https://cvc.cervantes.es/ensenanza/biblioteca_ele/carabela/pdf/47/47 053.pdf>. Acesso em: 5 nov. 2019.

SONG, Jeong-Weon. An investigation into the effects of an oral English diary using a voice bulletin board on English spoken performance. Multimedia-Assisted Language Learning, v. 12, n. 1, p. 125-150, 2009.

SPALACCI, Mirelle. Enfoque oral digital para el aula de francés LE con fines profesionales. Phonica, p. 222-229, 2014. Disponível em: <http://revistes.ub.edu/index.php/phonica/article/view/10983 >. Acesso em: 5 nov. 2019.

TOMÉ, Mario. Enseñanza y aprendizaje de la pronunciación de una lengua extranjera en al WEB 2.0. Revista de Lingüística y Lenguas Aplicadas, [S.1.], v. 5, p. 221-239, 2010. Disponível em: <http://dx.doi.org/10.4995/rlyla.2010.771>. Acesso em: 5 nov. 2019.

TORO, Juan. Enseñanza del español a través del cine hispano; marco teórico y ejemplo prácticos. MarcoELE. n. 8, p. 1-68, 2009. Disponível em: <https://marcoele.com/descargas/china/ji.toro_cinehispano.pdf $>$. Acesso em: 5 nov. 2019.

Recebido em: 10/06/2019

Aceito em: 22/10/2019

Title: Using video and blog digital tools to foster the learning of oral skills in Spanish as a Foreign Language

Título: $O$ uso de ferramentas digitais como vídeos e blogs para fomentar as habilidades orais em Espanhol como Língua Estrangeira 